Pacific Journal of Mathematics

ON COMPLETE AND INDEPENDENT SETS OF OPERATIONS 


\title{
ON COMPLETE AND INDEPENDENT SETS OF OPERATIONS IN FINITE ALGEBRAS
}

\author{
JeAn W. Butler
}

In [4] Post obtained a variety of results about truth functions in 2-valued sentential calculus. He studied sets of truth functions which could be used as primitive notions for various systems of 2-valued logics. In particular, he was interested in complete sets of truth functions, i.e., sets having the property that every truth function with an arbitrary finite number of arguments is definable in terms of the truth functions belonging to the set. Among other results Post established a computable criterion for a set of truth functions to be complete. Using this criterion he showed that there is a finite upper bound for the number of elements in any complete and independent set of primitive notions for the 2 -valued sentential calculus (and that actually the number 4 is the least upper bound). Alfred Tarski has asked to what extent these results can be extended to $n$-valued sentential calculus, for any finite $n$. It will be seen from this note that those results of Post concerning complete sets of truth functions can actually be extended. On the other hand it has been shown recently by A. Ehrenfeucht that the result concerning arbitrary sets of functions cannot be extended.

Both the results of Post and those of this note can be formulated in terms of truth functions of the 2 -valued ( $n$-valued) sentential calculus or in terms of finitary operations in arbitrary 2 element ( $n$ element) algebras. We choose the second alternative since the many-valued sentential calculi have a rather restricted significance in logic and mathematics.

Thus we shall concern ourselves with finitary operations under which a given set $A$ with $n$ elements is closed. For simplicity we restrict our attention to the case when $A$ is the set $N$ of all natural numbers less than $n$. This restriction implies no loss of generality, since all the results can be extended by isomorphism to any finite set with $n$ elements. For convenience we will identify $N$ with $n$, as is frequently done in modern set theory.

For any given natural number $k$, let $n^{k}$ be the set of all $k$-termed sequences $x=\left\langle x_{0}, x_{1}, \cdots, x_{k-1}\right\rangle$ with terms in $n$. Denote by $F_{n, k}$ the set of all $k$-ary operations on and to elements of $n$, i.e., of all function

Received December 15, 1959. This is the text of a talk given by the author at the Summer Institute of Symbolic Logic held in 1957 at Cornell University; it appeared in condensed form in "Summaries of talks presented at the Summer Institute of Symbolic Logic in 1957 at Cornell University" (mimeographed), 1957, pp. 78-80. The results were first communicated to the American Mathematical Society in [1]. The author thanks Professor Alfred Tarski for his guidance and encouragement. 
on $n^{k}$ to $n$. Let $F_{n}=\bigcup_{k<\infty} F_{n, k}$, i.e., $F_{n}$ is the set of all finitary operations on and to elements of $n$.

For any subset $X$ of $F_{n}$ we will denote by $\bar{X}$ the smallest set $Y$ which includes $X$ and satisfies the following conditions:

(i) if $f \in Y$ and $h$ is obtained from $f$ by exchanging or identifying two arguments, then $h \in Y$;

(ii) if $f \in Y, g \in Y$, and $h$ is obtained from $f$ by replacing an argument by $g$, then $h \in Y$. A function $f$ is said to be generated by a set $X$ if $f \in \bar{X}$. The set $X$ is called closed if $\bar{X}=X$, it is called complete if $\bar{X}=F_{n}$, it is called independent if there is no proper subset $X^{\prime}$ of $X$ for which $\bar{X}^{\prime}=\bar{X}$. A set $Y$ is called a basis for a set $X$ if $Y \subseteq X$ and $\bar{Y}=\bar{X}$. A function $f \in F_{n, c}$ is called reducible or reducible to first order if its values depend on at most one argument; i.e., if there is an $i<k$ and an $h \in F_{n, 1}$ such that for every sequence $x \in n^{k}$ we have $f(x)=h\left(x_{i}\right)$. Hence $f$ is not reducible if and only if for every $q<k$ there are $x, y \in n^{k}$ with $x_{q}=y_{q}$ and $f(x) \neq f(y)$. We will denote by $\mathscr{R}(f)$ the range of $f$. We single out two functions in $F_{n}$. $\mathbf{V}_{n}$ is the function of two arguments defined by the formula:

$$
x \bigvee_{n} y=\max (x, y) .
$$

$\sim_{n}$ is the function of one argument defined by:

$$
\sim_{n} x=x+1(\bmod n) \text {. }
$$

In the following few lemmas we will establish some properties of the notions just defined:

LEMma 1 . If $f \in F_{n, k}, n \geqq 3, f$ not reducible and $\mathscr{R}(f)=n$, then $\{f\} \cup F_{n, 1}$ generates a function $g \in F_{n, 2}, g$ not reducible and $\mathscr{R}(g)=n$.

Proof. We first establish that there exist $q<k$ and $u, v \in n^{k}$ such that $f(u) \neq f(v)$ and $u_{i}=v_{i}$ for all $i \neq q, i<n$. Since $\mathscr{R}(f)$ contains more than one element there exist $a, b \in n^{k}$ such that $f(a) \neq f(b)$. There are $k+1$ sequences $c^{(0)}, c^{(1)}, \cdots, c^{(k)} \in n^{k}$ with $c^{(0)}=a, c^{(k)}=b$, and such that for any $i<k, c^{(i+1)}$ is obtained from $c^{(i)}$ by replacing $c_{i}^{(i)}$ by $c_{i}^{(k)}$. Hence $c^{(i)}$ and $c_{i}^{(i+1)}$ differ only in the $i$ th coordinate, moreover $c_{i}^{(j)}=a_{i}$ for $i \geqq j$ and $c_{i}^{(j)}=b_{i}$ for $i<j$. Since $f(a)=f\left(c^{(0)}\right)=$ $f\left(c_{0}^{(0)}, c_{1}^{(0)}, \cdots, c_{k-1}^{(0)}\right), f(b)=f\left(c^{(k)}\right)$ and $f(a) \neq f(b)$ it cannot be the case that $f\left(c^{(i)}\right)=f\left(c^{(i+1)}\right)$ for all $i<k$. Therefore there is some $q<k$ such that $f\left(c^{(q)}\right) \neq f\left(c^{(q+1)}\right)$ and $c_{i}^{(q)}=c_{i}^{(q+1)}$ for all $i \neq q, i<k$. Take $c^{(q)}$ for $u$ and $c^{(q+3)}$ for $v$.

Since $\mathscr{R}(f)=n$, we can choose $n$ sequences $y^{(0)}, y^{(1)}, \cdots, y^{(n-1)} \in n^{k}$ such that $f\left(y^{(0)}\right)=f(u), f\left(y^{(1)}\right)=f(v)$, and each value in $n$ is taken on by $f$ for some $y^{(i)}$. There also exist $w, z \in n^{k}$ for which $w_{q}=z_{q}$ and 
$f(w) \neq f(z)$, since if this were not the case $f$ would depend only on its $q$ th argument and thus be reducible.

There are two possible cases: (i) there exist $w, z \in n^{k}$, with $w_{q}=z_{q}$, and $f(w) \neq f(z), f(w) \neq f(u), f(w) \neq f(v)$; (ii) for every $w, z \in n^{k}$ with $w_{q}=z_{q}$ and $f(w) \neq f(z)$ neither $f(w)$ nor $f(z)$ is different from both $f(u)$ and $f(v)$.

If case (i) holds there exist $w, z \in n^{k}$ for which $w_{q}=z_{q}$, and $f(w) \neq f(z), f(w) \neq f(u), f(w) \neq f(v)$. With no loss of generality we may assume $f\left(y^{(2)}\right)=f(w)$. We define $k$ functions $h_{0}, h_{1}, \cdots, h_{k-1} \in F_{n, 1}$ separately for $h_{q}$ and for $h_{j}, j \neq q$,

$$
h_{q}(x)=\left\{\begin{array}{ll}
u_{q} & x=0 \\
v_{q} & x=1 \\
z_{q} & x=2 \\
y_{q}^{(x)} & x>2
\end{array} \quad h_{\jmath}(x)= \begin{cases}u_{j} & x=0 \\
z_{j} & x=1 \\
w_{j} & x=2 \\
y_{j}^{(x)} & x>2 .\end{cases}\right.
$$

We define $g \in F_{n, 2}$ as follows:

$$
g(x, y)=f\left(h_{0}(x), h_{1}(x), \cdots, h_{q-1}(x), h_{q}(y), h_{q+1}(x), \cdots, h_{k-1}(x)\right) .
$$

Notice that $y$ appears only in the $q$ th coordinate of $f . \mathscr{R}(g)=n$ since $g(0,0)=f(u)=f\left(y^{(0)}\right), g(0,1)=f(v)=f\left(y^{(1)}\right), g(2,2)=f(w)=f\left(y^{(2)}\right)$, and $g(i, i)=f\left(y^{(i)}\right)$ for $i>2$. Moreover $g$ is not reducible, since $g(0,0) \neq$ $g(0,1)$ and $g(1,2) \neq g(2,2)$.

If case (ii) holds we take for $h_{q}$ the identity function in $F_{n, 1}$ and using any $w, z \in n^{k}$ for which $w_{q}=z_{q}$ and $f(w) \neq f(z)$ we choose $k-1$ functions $h_{j} \in F_{n, 1}$ for $j \neq q$, satisfying

$$
h_{j}(x)= \begin{cases}u_{j} & x=0 \\ w_{j} & x=1 \\ z_{j} & x=2\end{cases}
$$

and then define $g$ by (1). Now for any $s \in n^{k}$ with $s_{q}=y_{q}^{(i)}, 2<i \leqq n$, condition (ii) guarantees that $f(s)=f\left(y^{(i)}\right)$ since $f\left(y^{(i)}\right) \neq f(u)$ and $f\left(y^{(i)}\right) \neq f(v)$. Therefore $g\left(0, u_{q}\right)=f(u)=f\left(y^{(0)}\right), g\left(1, v_{q}\right)=f(v)=f\left(y^{(1)}\right)$, $g\left(m, y_{q}^{(i)}\right)=f\left(y^{(i)}\right)$ for $m<n$ and $2 \leqq i<n$. Hence $\mathscr{R}(g)=n$. The function $g$ is not reducible, since $g\left(0, u_{q}\right) \neq g\left(0, v_{q}\right)$ and $g\left(1, w_{q}\right) \neq g\left(2, w_{q}\right)$.

LEMMA 2. If $f \in F_{n, 2}$ is not reducible and $\mathscr{R}(f)$ has $p$ elements $p \geqq 3$, then there exist $i, j, k, l<n$ such that among the function values $f(i, k), f(i, l), f(j, k), f(j, l)$ at least three distinct values are represented.

Proof. There are two possibilities;

(i) in the table of $f$ all value in $\mathscr{R}(f)$ are taken on across some row, i.e., there is an $i<n$ such that the set of all values $f(i, j)$ with 
$j<n$ coincides with $\mathscr{R}(f)$;

(ii) in no row are all values in $\mathscr{R}(f)$ taken on.

If (i) holds, since $f$ is not reducible there must be $j, l<n$ for which $f(i, l) \neq f(j, l)$. Since $p \geqq 3$ and all values in $\mathscr{R}(f)$ are taken on in the $i$ th row there must be a $k<n$ such that $f(i, k)$ is distinct from both $f(i, l)$ and $f(j, l)$.

If (ii) holds, since $f$ is not reducible there is some non-constant row, i.e., some $i, j^{\prime}, j^{\prime \prime}$ such that $f\left(i, j^{\prime}\right) \neq f\left(i, j^{\prime \prime}\right)$. However, by assumption there is a $w \in \mathscr{R}(f)$ which does not appear in this row, i.e., for all $x<p, f(i, x) \neq w$. Since $w \in \mathscr{R}(f)$ there are $j, l<n$ for which $f(j, l)=w$. Hence $f(i, l) \neq f(j, l)$. Since $w$ does not appear in the $i$ th row and the $i$ th row is not constant there is some $k<n$ such that the value $f(i, k)$ is different from both $f(i, l)$ and $f(j, l)$.

LEMmA 3. If $f \in F_{n, 2}$ is not reducible and $\mathscr{R}(f)$ has exactly $p$ elements, $3 \leqq p \leqq n$, then there exist two functions $h_{1}, h_{2} \in F_{n, 1}$ with $\mathscr{R}\left(h_{1}\right), \mathscr{R}\left(h_{2}\right)$ each consisting of at most $p-1$ elements, and such that for every $x \in \mathscr{R}(f)$ we have $f\left(h_{1}(x), h_{2}(x)\right)=x$.

Proof. By Lemma 2 we can find $i, j, k, l<n$ such that $f(i, k)$, $f(i, l), f(j, k), f(j, l)$ represent at least three distinct values. Assume $f(i, k)=u, f(i, l)=v, f(j, k)=w$ are all different. Functions $h_{1}, h_{2} \in$ $F_{n, 1}$ can be found such that

$$
\begin{aligned}
h_{1}(u) & =i & h_{2}(u) & =k \\
h_{1}(v) & =i & h_{2}(v) & =l \\
h_{1}(w) & =j & h_{2}(w) & =k
\end{aligned}
$$

and

$$
\begin{gathered}
h_{1}(x)=i, \quad h_{2}(x)=k \text { for } x \notin \mathscr{R}(f) \\
f\left(h_{1}(x), h_{2}(x)\right)=x \text { for } x \in \mathscr{R}(f) \sim\{u, v, w\} .
\end{gathered}
$$

Clearly, $h_{1}$ and $h_{2}$ satisfy the requirements of the Lemma. The proof in the other cases is analogous.

LEMmA 4. If $f \in F_{n, 2}$ and $2 \leqq p \leqq n$, and there exist $i, j, k<n$ such that for all $y<p$

$$
f(i, y)=y \text { and } f(j, y)=k
$$

then $f$ together with the functions in $F_{n, 1}$ generate a function $g \in F_{n, 2}$ such that $g(x, y)=x \bigvee_{n} y$ for $x, y<p$.

Proof. We shall prove, by induction on $p$, a slightly weakened form of the theorem, replacing the condition $i, j, k<n$ by $i, j, k<p$. The 
theorem as stated then follows, since $\{f\} \cup F_{n, 1}$ generates a function satisfying the strengthened hypothesis.

For $p=2$, since $i, j<p$ the function $f$ must agree on $\{0,1\}$ with one of the following four tables:
(i) \begin{tabular}{l|ll} 
& 0 & 1 \\
\hline 0 & 0 & 1 \\
1 & 1 & 1
\end{tabular}
(ii) \begin{tabular}{l|ll} 
& 0 & 1 \\
\hline & 0 & 1 \\
1 & 0 & 0
\end{tabular}
(iii) \begin{tabular}{l|ll} 
& 0 & 1 \\
\hline 0 & 0 & 0 \\
1 & 0 & 1
\end{tabular}

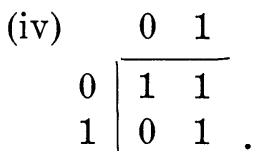

In case (i) $f$ itself may be taken for $g$. In the other cases using any homomorphism $h \in F_{n, 1}$ which exchanges 0 and $1, \mathrm{~g}$ may be constructed as (ii) $h(f(x, h(y)))$, (iii) $h(f(h(x), h(y)))$, (iv) $f(h(x), y)$.

Assume the theorem is true for $p-1$. From $\{f\} \cup F_{n, 1}$ we can construct a function satisfying the induction hypothesis for $p-1$. Thus we can generate a function $g^{\prime}$ such that

$$
g^{\prime}(x, y)=x \mathrm{~V}_{n} y \text { for } x, y<p-1 .
$$

Now choose functions $h_{1}, h_{2} \in F_{n, 1}$ such that

$$
h_{1}(x)=\left\{\begin{array}{ll}
i & \text { if } x<p-1 \\
j & \text { if } x=p-1
\end{array} \quad h_{2}(x)= \begin{cases}p-1 & \text { if } x=k \\
k & \text { if } x=p-1 \\
x & \text { otherwise }\end{cases}\right.
$$

and construct $f^{\prime} \in F_{n, 2}$ as follows:

$$
f^{\prime}(x, y)=h_{2}\left(f\left(h_{1}(x), h_{2}(y)\right)\right) .
$$

It can be seen that

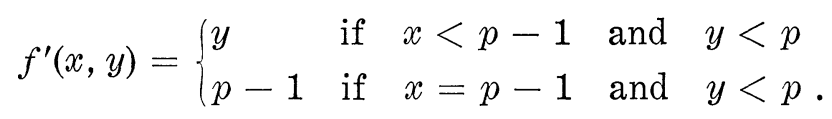

Now we define $g \in F_{n, 2}$ :

$$
g(x, y)=f^{\prime}\left(f^{\prime}(x, y), g^{\prime}(x, y)\right) .
$$

For $\quad x, y<p-1, \quad g(x, y)=g^{\prime}(x, y)=x \bigvee_{n} y ; \quad$ for $\quad x=p-1, \quad y<p$, $g(x, y)=f^{\prime}\left(p-1, g^{\prime}(p-1, y)\right)=p-1 ;$ for $x<p-1$ and $y=p-1$, $g(x, y)=f^{\prime}\left(p-1, g^{\prime}(x, p-1)\right)=p-1$. Therefore $g$ agrees with $\mathbf{V}_{n}$ for $x, y<p$, which estalishes the Lemma.

Lemma 5. If $f \in F_{n, 2}$ is not reducible and $\mathscr{R}(f)=p, 3 \leqq p \leqq n$, then $f$ together with the functions in $F_{n, 1}$ generate a function $g \in F_{n, 2}$ such that $g(x, y)=x \bigvee_{n} y$ for all $x, y<p$.

Proof. The proof is by induction on $p$. For $p=3$, using the $i, j$, $k, l$ of Lemma 3 and appropriate homomorphisms from $F_{n, 1}$ we can 
generate a function $h \in F_{n, 2}$ such that the values of $h$ on $\{0,1\}$ agree with one of the two tables:
(i) \begin{tabular}{r|rr} 
& 0 & 1 \\
\cline { 2 - 3 } & 0 & 1 \\
1 & 2 & 2
\end{tabular}

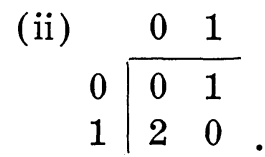

We then choose five functions $h_{1}, h_{2}, h_{3}, h_{4}, h_{5} \in F_{n, 1}$ such that

$$
\begin{array}{lllll}
h_{1}(0)=0 & h_{2}(0)=0 & h_{3}(0)=0 & h_{4}(0)=0 & h_{5}(0)=0 \\
h_{1}(1)=0 & h_{2}(1)=1 & h_{3}(1)=0 & h_{4}(1)=1 & h_{5}(1)=2 \\
h_{1}(2)=1 & h_{2}(2)=1 & h_{3}(2)=0 & h_{4}(2)=0 & h_{5}(2)=2 .
\end{array}
$$

In case (i) $g$ can be constructed as

$$
g(x, y)=h\left(h\left(x, h_{1}(y)\right), h\left(x, h_{2}(y)\right)\right) .
$$

To construct $g$ in case (ii) we define $g^{\prime}, g^{\prime \prime} \in F_{n, 2}$

$$
\begin{aligned}
g^{\prime}(x, y) & =h\left(h\left(h_{3}(x), h_{1}(y)\right), h_{2}\left(h\left(h_{1}(x), h_{4}(y)\right)\right)\right) \\
g^{\prime \prime}(x, y) & =h_{4}\left(g^{\prime}\left(x, h_{5}(y)\right)\right)
\end{aligned}
$$

and then

$$
g(x, y)=g^{\prime}\left(g^{\prime \prime}(x, y), g^{\prime}(x, y)\right) .
$$

Assuming the theorem true for $p-1$, we prove it for $p, 3<p \leqq n$. First we construct a function $f^{\prime \prime}$ satisfying the induction hypothesis for $p-1$. To do this we apply Lemma 2, taking an $i, j, k, l$ such that $f(i, k), f(i, l), f(j, k), f(j, l)$ represent at least 3 distinct values, $u, v, w$. Since $p>3$ there is a value $z \in \mathscr{R}(f) \sim\{u, v, w\}$. Let $h \in F_{n, 1}$ such that

$$
h(x)= \begin{cases}u & x=z \\ x & \text { otherwise. }\end{cases}
$$

Then the function $h(f(x, y))$ is not reducible and has $p-1$ elements in its range. The application of an appropriate isomorphism from $F_{n, 2}$ will produce a function $f^{\prime \prime} \in F_{n, 2}$ with $\mathscr{R}\left(f^{\prime \prime}\right)=p-1$ and $f^{\prime \prime}$ not reducible. Then by the induction assumption we can generate a function $g^{\prime \prime} \in F_{n, 2}$ such that

$$
g^{\prime \prime}(x, y)=x \bigvee_{n} y \quad \text { for } \quad x, y<p-1 .
$$

Next by Lemma 3 there exist $h_{1}, h_{2} \in F_{n, 1}$ with $\mathscr{R}\left(h_{1}\right), \mathscr{R}\left(h_{2}\right)$ each consisting of at most $p-1$ elements and such that

$$
f\left(h_{1}(x), h_{2}(x)\right)=x
$$

for $x<p$. 
Let $h_{3}, h_{4} \in F_{n, 1}$ be isomorphisms such that

$$
\begin{aligned}
& h_{3}(x)<p-1 \text { for } x \in \mathscr{R}\left(h_{1}\right) \\
& h_{4}(x)<p-1 \text { for } x \in \mathscr{R}\left(h_{2}\right),
\end{aligned}
$$

and define $f^{\prime} \in F_{n, 2}, h_{5}, h_{6} \in F_{n, 1}$ :

$$
\begin{array}{cl}
h_{5}(x)=h_{3}\left(h_{1}(x)\right), & x<n \\
h_{6}(x)=h_{4}\left(h_{2}(x)\right), & x<n \\
f^{\prime}(x, y)=f\left(h_{3}^{-1}(x), h_{4}^{-1}(y)\right), & x, y<n .
\end{array}
$$

Then

$$
f^{\prime}\left(h_{5}(x), h_{6}(x)\right)=x \quad \text { for } \quad x<p
$$

and $\mathscr{R}\left(h_{5}\right), \mathscr{R}\left(h_{6}\right) \subseteq p-1$.

We can now define a function $g^{\prime} \in F_{n, 2}$ as follows:

$$
g^{\prime}(x, y)=f^{\prime}\left(g^{\prime \prime}\left(x, h_{1}(y)\right), g^{\prime \prime}\left(x, h_{2}(y)\right)\right) \text {. }
$$

Then

$$
g^{\prime}(0, y)=f^{\prime}\left(h_{5}(y), h_{6}(y)\right)=y \quad \text { for } \quad y<p
$$

and

$$
g^{\prime}(p-2, y)=f^{\prime}(p-2, p-2) \text { for } y<p .
$$

Therefore by Lemma 4 we can generate a function $g \in F_{n, 2}$ such that $g(x, y)=x \bigvee_{n} y$ for $x, y<p$.

Lemma 6. If $f \in F_{n}, n \geqq 3, f$ is not reducible, and $\mathscr{R}(f)=n$ then $F_{n, 1} \cup\{f\}$ is complete.

Proof. By definition $\sim_{n} \in F_{n, 1}$. By Lemma 1 there is a $g \in$ $\overline{F_{n, 1} \cup\{f\}} \cap F_{n, 2}$ such that $\mathscr{R}(g)=n$ and $g$ is not reducible. Using Lemma 5 with $p=n$ we see that $\mathrm{V}_{n} \in \overline{F_{n, 1} \cup\{f\}}$. It is known ${ }^{1}$ that the set $\left\{\mathbf{V}_{n}, \sim_{n}\right\}$ is complete. Clearly, if $X \subseteq \bar{Y}$ and $X$ is complete then $Y$ is complete. Therefore $F_{n, 1} \cup\{f\}$ is complete.

In [4] Post established a necessary and sufficient condition for a set $X \subseteq F_{2}$ to be complete. In order to extend this result to $n>2$ we use his method. This consists in constructing a finite family $\mathscr{C}_{n}$ of proper closed subsets of $F_{n}$ satisfying the condition that every proper closed subset of $F_{n}$ is included in some set of the family. The existence of such a finite family of maximal sets is an important property of the lattice of all closed subsets of $F_{n}$.

By our definition $F_{n, 1}$ is closed. Moreover $F_{n, 1}$ is finite, containing

1 Post [3]. 
exactly $n^{n}$ elements; therefore the family of all closed subsets of $F_{n, 1}$ is finite. For each closed $S \subseteq F_{n, 1}, n \geqq 3$, we define a set $M(S)$ as follows:

(i) if $S=F_{n, 1}$ then $M(S)$ is the set of all functions $f \in F_{n}$ such that either $f$ is reducible or $\mathscr{R}(f)$ is a proper subset of $n$;

(ii) if $S \neq F_{n, 1}$, then $M(S)$ is the set of all functions $f \in F_{n}$ satisfying the following condition: if in $f$ we replace zero or more arguments by functions in $S$ and then identify all arguments, the resulting function is in $S$.

Finally, we take as $\mathscr{I}_{n}, n \leqq 3$, the family of all sets $M(S)$ where $S$ is any closed subset of $F_{n, 1}{ }^{2}$ That this family $\mathscr{M}_{n}$ actually has the property mentioned above is seen from the following.

Lemma 7. Let $S$ is a closed subset of $F_{n, 1},(n \geqq 3)$. Then

(i) $M(S)$ is closed.

(ii) $M(S)$ is a proper subset of $F_{n}$.

(iii) $M(S) \cap F_{n, 1}=S$.

(iv) If $Y$ is a proper closed subset of $F_{n}$ with $Y \cap F_{n, 1}=S$ then $Y \subseteq M(S)$.

Proof. We establish Lemma 7 first for the case $S \neq F_{n, 1} \cdot M(S)$ is closed, since the defining property for $M(S)$ is preserved under exchange or identification of variables and also under substitution. Since $S$ is a proper subset of $F_{n, 1}, M(S)$ does not contain all functions of $F_{n, 1}$. Therefore $M(S)$ is a proper subset of $F_{n} . \quad S \subseteq M(S) \cap F_{n, 1} \subseteq S$, hence $M(S) \cap F_{n, 1}=S$. The fourth property can be verified directly from the definition of $M(S)$ : Let $Y$ be any proper closed subset of $F_{n}$ with $Y \cap F_{n, 1}=S, f \in Y$, and $h$ a function obtained by replacing zero or more arguments of $f$ by functions in $S$ and then identifying all arguments. Since $S \subseteq Y$ and $Y$ is closed, $h \in Y$. But $Y \cap F_{n, 1} \subseteq S$ and $h \in F_{n, 1}$, so $h \in S$. Thus every function $f \in Y$ is in $M(S)$. Therefore $Y \subseteq M(S)$.

We turn now to the case $S=F_{n, 1}$. That $M\left(F_{n, 1}\right)$ is closed follows from its definition: Both reducibility and range different from $n$ are preserved under exchange or identification of variables. Let $f, g \in M\left(F_{n, 1}\right)$, $h$ a function obtained by replacing an argument of $f$ by the function $g$. If $\mathscr{R}(g) \neq n$ then $\mathscr{R}(h) \neq n$ and $h \in M\left(F_{n, 1}\right)$. If $g$ is reducible, either $h$ is reducible or $h=f$, so $h \in M\left(F_{n, 1}\right)$. Clearly $M\left(F_{n, 1}\right)$ is a proper subset of $F_{n}$ since there exist functions in $F_{n}$ with full range $n$ which are not reducible. $M\left(F_{n, 1}\right) \cap F_{n, 1}=F_{n, 1}$, since every function in $F_{n, 1}$ is reducible. The proof of the fourth property follows from Lemma 6:

2 The corresponding family for $n=2$ contains nine elements since $F_{2,1}$ has exactly nine closed subsets. In [4] Post defined these nine sets individually: $C_{4}, R_{2}, R_{3}, R_{9}, C_{2}, C_{3}, D_{3}$, $A_{1}, L_{1}$. Our definition of $M(S)$ is directly applicable to the eight proper closed subsets of $F_{2,1}$. However, it is of interest to note that in the case $S=F_{n, 1}$ the structure of $M(S)$ is essentially different for $n>2$. 
Let $Y$ be any proper closed subset of $F_{n}$ with $Y \cap F_{n, 1}=F_{n, 1}$. Clearly, $Y$ cannot be complete. By Lemma 6 , since $F_{n, 1} \subseteq Y, Y$ cannot contain any function $f$, with $\mathscr{R}(f)=n$, which is not reducible. Hence $Y \cong$ $M\left(F_{n, 1}\right)$. This completes the proof.

Thus we see that the family $\mathscr{C}_{n}$ of all sets $M(S)$ where $S$ is any closed subset of $F_{n, 1}$ consists of finitely many proper closed subsets of $F_{n}$. Moreover, if $X$ is any proper closed subset of $F_{n}$ by property (iv) of Lemma $7 X \subseteq M\left(X \cap F_{n, 1}\right) \in \mathscr{I}_{n}$, since $X \cap F_{n, 1}$ is a closed subset of $F_{n, 1}$.

We now state the main result of the note:

THEOREM 1. A necessary and sufficient condition for a set $X \subseteq F_{n}$, $n \geqq 3$, to be complete is that for every closed subset $S$ of $F_{1, n}$ there is an $f \in X$ such that $f \notin M(S) .^{3}$

The proof of this theorem follows directly from Lemma 7. If there were any closed subset $S$ of $F_{1, n}$ such that $X \subseteq M(S)$ then $\bar{X} \subseteq M(S)$ since $M(S)$ is closed and hence $X$ would not be complete. On the other hand if for every closed subset $S$ of $F_{1, n}$ there is an $f \in X \sim M(S)$ then $\bar{X} \nsubseteq M(S)$ for any closed subset $S$ of $F_{1, n}$. By Lemma $7, \bar{X}$ cannot be a proper subset of $F_{n}$. Therefore $X$ must be complete.

COROLlary 1. A set $X \subseteq F_{n}$ is complete if and only if $F_{n, 1} \subseteq \bar{X}$, $n \geqq 3$, and there is an $f \in X$ such that $\mathscr{R}(f)=n$, and $f$ is not reducible.

Proof. If $X$ is complete then $F_{n, 1} \subseteq \bar{X}$; and by Theorem $1, X \nsubseteq$ $M\left(F_{n, 1}\right)$. Therefore there is an $f \in X \sim M\left(F_{n, 1}\right)$; i.e., $f \in X, \mathscr{R}(f)=n$, and $f$ not reducible. On the other hand if $F_{n, 1} \subseteq \bar{X}$, then $X$ is not included in $M(S)$ for any proper closed subset $S$ of $F_{n, 1}$. If, in addition, there is an $f \in X$ with $\mathscr{R}(f)=n$ and $f$ not reducible, then $X \nsubseteq M\left(F_{n, 1}\right)$. Therefore by Theorem $1, X$ must be complete.

We now state two further results, Theorems 2 and $3,{ }^{4}$ which follow easily from Theorem 1;

THEOREM 2. There exist finite decision procedures to determine

3 An analogous result for $n=2$ with the family $\mathscr{k}_{n}$ replaced by the set $\left\{D_{3}, C_{2}, C_{3}, A_{1}, L_{1}\right\}$ was obtained by Post in [4]. It may be noted that our theorem can be sharpened to include this result by adding the restriction: $S$ contains the identity function and at least one other element.

4 Yablonskii in [5] states without proof Theorem 2, which he attributes to A. V. Kuznecov. He also attributes to Kuznecov another result which he states (again without proof) as follows: Every complete set (included in $F_{n}$ ) contains a finite complete subset (i.e., a finite basis). In this form the result is rather obvious and follows directly from the results of Post [3]; compare the first part of the proof of Corollary 2. The subsequent remarks of Yablonskii make it likely, however, that Kuznecov obtained a stronger result established here as Corollary 2. 
whether or not any finite subset of $F_{n}, n \geqq 3$, is complete and if complete whether independent. ${ }^{5}$

This theorem depends essentially on the computable character of our definition of the sets $M(S)$. This means that for any $f \in F_{n}$ and any closed subset $S$ of $F_{n, 1}$ we can tell by a finite procedure whether or not $f \in M(S)$. Therefore if $X$ is a finite set, Theorem 1 provides a finite method for determining whether or not $X$ is complete. This part of the proof can also be based on Corollary 1 . The only thing to be shown is that all functions belonging to $F_{n, 1} \cap \bar{X}$ can be obtained by means of a well determined finite procedure.

If $X$ is complete then $X$ is independent if and only if no proper subset of $X$ is complete. For a finite complete $X$, therefore, we can determine in finitely many steps whether or not $X$ is independent.

THEOREM 3. For any natural number $n, n \geqq 2$, there is a natural number $p$ such that every complete and independent subset of $F_{n}$ has at most $p$ elements. ${ }^{6}$

For $n=2$ this theorem was proved by Post in [4]. For $n \geqq 3$ it can be derived directly from Theorem 1 . Let $p$ be the number of elements in the family $\mathscr{M}_{n}$ of all sets $M(S)$ for $S$ any proper closed subset of $F_{n, 1}$. By Theorem 1, any set which contains an $f \notin M(S)$ for each $M(S)$ in $\mathscr{A}_{n}$ is complete. Thus any complete set with more than $p$ elements would contain a proper subset which is complete.

COROLlaRY 2. For any number $n, n \geqq 2$, there is a natural number $p$ such that every complete set included in $F_{n}$ has a finite basis with at most $p$ element.

If $X F_{n} \subseteq$ is complete, then $\bigvee_{n}, \sim_{n} \in \bar{X}$. Hence $\left\{\bigvee_{n}, \sim_{n}\right\}$ can be generated by a finite subset $Y$ of $X$. Since $\left\{V_{n}, \sim_{n}\right\}$ is complete $Y$ must be complete. Let $Z$ be any complete independent subset of $Y$. $Z$ is a finite basis of $X$ and by Theorem 3, $Z$ has at most $p$ elements.

By modifying the proof of this result (and in fact making the argument independent of Theorem 1) A. Tarski has obtained the following generalization of Theorem 3 .

THEOREM 4. For any closed set $X \subseteq F_{n}$ which has a finite basis there is a natural number $q$ such that every independent basis of $X$ has at most $q$ elements.

The method of proof is similar to the proof of Theorem 3. We replace the family $\mathscr{M}_{n}$ by a finite family $\mathscr{L}_{X}=\left\{L_{0}, L_{1}, \cdots, L_{q-1}\right\}$ of closed proper subsets of $X$ with the property that for any closed proper

${ }^{5} \mathrm{By}$ Post's results in [4], this theorem is also valid for $n=2$ since the conditions defining the sets $D_{3}, C_{2}, C_{3}, A_{1}$ and $L_{1}$ are finitely computable.

${ }^{6}$ For $n=3$, Yablonskii in [5] found $p=18$. 
subset $Y$ of $X$ there is 'a set $L_{i}, i<q$, in $\mathscr{L}_{X}$ such that $Y \cong L_{i}$. $\mathscr{L}_{X}$ is constructed as follows. Let $B$ be any finite basis of $X$. Since $B$ is finite there is a natural number $k$ such that $B \cong \bigcup_{i<k} F_{i, n}$. For each $A$ satisfying

$$
A \subseteq X \cap \bigcup_{i<k} F_{n, i}, \quad A \neq X, \text { and } \bar{A} \cap \bigcup_{i<k} F_{n, i}=A
$$

we define the set $L(A)$ in the same manner as the sets $M(S)$ were defined for $S \neq F_{n, 1}$. The proof that the sets $L(A)$ are closed proper sets with the required property in the lattice all closed subsets of $X$ is entirely analogous to the proof of Lemma 7 .

For complete sets the upper bound $q$ of Theorem 4 is much larger than the $p$ of Theorem 3 .

For $n=2$, Post in [4] computed an upper bound $p=5$ in Theorem 3 , and then showed that actually 4 was the least upper bound. He also proved that every closed subset of $F_{2}$ has a finite basis. Therefore, two further questions arise for $n \geqq 3$ :

(1) does every closed system of functions in $F_{n}$ have a finite basis;

(2) (proposed by A. Tarski) is there any finite procedure to determine the least upper bound for the number of elements in any independent basis of the complete system $F_{n}$.

The solutions of these two problems have been communicated to me by A. Ehrenfeucht. He has shown that the solution of problem (2) is positive, while that of problem (1) is negative. Ehrenfeucht exhibits a very simple closed subset of $F_{n}, n \geqq 3$, which has no finite basis.

(Added in proof.) It has been communicated to me by Professor C. C. Chang that Lemma 5 was obtained by Jerzy Slupecki in "A criterion of fullness of many-valued systems of propositional logic", Comptes Rendues des séances de la Société des Sciences et des lettres de Varsovie 33, 1939, Classe III, pp 102-109. Slupecki proves the following extension of Lemma 5: If $F_{n, 1} \subseteq X$ then $X$ is complete if and only if there is an $f \in X, f \in F_{n, 2}, f$ not reducible and $\mathscr{R}(f)=n$. Note that Lemma 6 and Corollary 1 extend this result by using Lemmas 1 and 7 to remove the condition $f \in F_{n, 2}$, which is necessary for the main results of this note.

\section{BIBLIOGRAPHY}

1. Jean W. Butler, On operations in finite algebras, Bull. Amer. Math. Soc., 62 (1956), Abstract 552.

2. A. Ehrenfeucht, review of [5], Journal of Symbolic Logic 20 (1955), 175.

3. E. L. Post, Introduction to a general theory of elementary propositions, American Journal of Mathematics, 43 (1921), 163-185.

4. E. L. Post, The two-valued interative systems of mathematical logic, Princeton Annals of Math. Studies, 5 (1941).

5. S. V. Yablonskii (S. V. Áblonskij), On functional completeness in the three-valued calculus, Doklady Akademii Nauk SSSR, 95 (1954), 1153-1155. 



\section{PACIFIC JOURNAL OF MATHEMATICS}

\section{EDITORS}

David GILbarg

Stanford University

Stanford, California

\section{F. H. BRowneLL}

University of Washington

Seattle 5, Washington

\section{A. L. Whiteman}

University of Southern California Los Angeles 7, California

\section{J. PAIGe}

University of California

Los Angeles 24, California

\section{ASSOCIATE EDITORS}
E. F. BECKENBACH
T. M. CHERRY
D. DERRY

\author{
E. HEWITT \\ A. HORN \\ L. NACHBIN
}

\author{
M. OHTSUKA \\ H. L. ROYDEN \\ M. M. SCHIFFER
}

E. SPANIER

E. G. STRAUS

F. WOLF

\section{SUPPORTING INSTITUTIONS}

\author{
UNIVERSITY OF BRITISH COLUMBIA \\ CALIFORNIA INSTITUTE OF TECHNOLOGY \\ UNIVERSITY OF CALIFORNIA \\ MONTANA STATE UNIVERSITY \\ UNIVERSITY OF NEVADA \\ NEW MEXICO STATE UNIVERSITY \\ OREGON STATE COLLEGE \\ UNIVERSITY OF OREGON \\ OSAKA UNIVERSITY \\ UNIVERSITY OF SOUTHERN CALIFORNIA
}

\author{
STANFORD UNIVERSITY \\ UNIVERSITY OF TOKYO \\ UNIVERSITY OF UTAH \\ WASHINGTON STATE COLLEGE \\ UNIVERSITY OF WASHINGTON \\ AMERICAN MATHEMATICAL SOCIETY \\ CALIFORNIA RESEARCH CORPORATION \\ HUGHES AIRCRAFT COMPANY \\ SPACE TECHNOLOGY LABORATORIES \\ NAVAL ORDNANCE TEST STATION
}

\footnotetext{
Mathematical papers intended for publication in the Pacific Journal of Mathematics should be typewritten (double spaced), and the author should keep a complete copy. Manuscripts may be sent to any one of the four editors. All other communications to the editors should be addressed to the managing editor, L. J. Paige at the University of California, Los Angeles 24, California.

50 reprints per author of each article are furnished free of charge; additional copies may be obtained at cost in multiples of 50 .
}

The Pacific Journal of Mathematics is published quarterly, in March, June, September, and December. The price per volume (4 numbers) is $\$ 12.00$; single issues, $\$ 3.50$. Back numbers are available. Special price to individual faculty members of supporting institutions and to individual members of the American Mathematical Society: $\$ 4.00$ per volume; single issues, $\$ 1.25$.

Subscriptions, orders for back numbers, and changes of address should be sent to Pacific Journal of Mathematics, 2120 Oxford Street, Berkeley 4, California.

Printed at Kokusai Bunken Insatsusha (International Academic Printing Co., Ltd.), No. 6, 2-chome, Fujimi-cho, Chiyoda-ku, Tokyo, Japan.

PUBLISHED BY PACIFIC JOURNAL OF MATHEMATICS, A NON-PROFIT CORPORATION

The Supporting Institutions listed above contribute to the cost of publication of this Journal, but they are not owners or publishers and have no responsibility for its content or policies. 


\section{Pacific Journal of Mathematics}

\section{Vol. 10, No. $4 \quad$ December, 1960}

M. Altman, An optimum cubically convergent iterative method of inverting a linear bounded operator in Hilbert space . . . . . . . . . . . . . . . . . . . . . . . . . . 1107

Nesmith Cornett Ankeny, Criterion for rth power residuacity ................. 1115

Julius Rubin Blum and David Lee Hanson, On invariant probability measures I . . . . . 1125

Frank Featherstone Bonsall, Positive operators compact in an auxiliary topology ..... 1131

Billy Joe Boyer, Summability of derived conjugate series . . . . . . . . . . . . . . . . 1139

Delmar L. Boyer, A note on a problem of Fuchs . . . . . . . . . . . . . . . . . 1147

Hans-Joachim Bremermann, The envelopes of holomorphy of tube domains in infinite

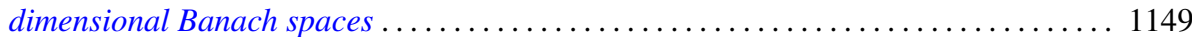

Andrew Michael Bruckner, Minimal superadditive extensions of superadditive

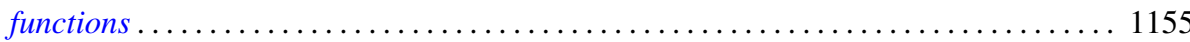

Billy Finney Bryant, On expansive homeomorphisms .................... 1163

Jean W. Butler, On complete and independent sets of operations in finite algebras . . . . . 1169

Lucien Le Cam, An approximation theorem for the Poisson binomial distribution ...... 1181

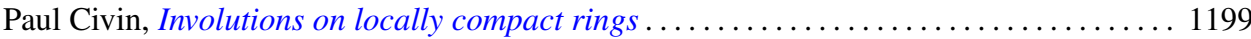

Earl A. Coddington, Normal extensions of formally normal operators . . . . . . . . . 1203

Jacob Feldman, Some classes of equivalent Gaussian processes on an interval ........ 1211

Shaul Foguel, Weak and strong convergence for Markov processes . . . . . . . . . . . 1221

Martin Fox, Some zero sum two-person games with moves in the unit interval ........ 1235

Robert Pertsch Gilbert, Singularities of three-dimensional harmonic functions . . . . . . . 1243

Branko Grünbaum, Partitions of mass-distributions and of convex bodies by

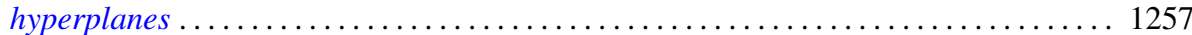

Sidney Morris Harmon, Regular covering surfaces of Riemann surfaces ........... 1263

Edwin Hewitt and Herbert S. Zuckerman, The multiplicative semigroup of integers

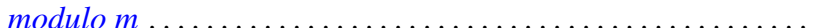

Paul Daniel Hill, Relation of a direct limit group to associated vector groups . ......... 1309

Calvin Virgil Holmes, Commutator groups of monomial groups . .

James Fredrik Jakobsen and W. R. Utz, The non-existence of expansive homeomorphisms

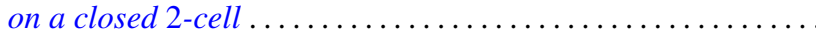

John William Jewett, Multiplication on classes of pseudo-analytic functions . . . . . . . 1323

Helmut Klingen, Analytic automorphisms of bounded symmetric complex domains . . . . 1327

Robert Jacob Koch, Ordered semigroups in partially ordered semigroups . . . . . . . . 1333

Marvin David Marcus and N. A. Khan, On a commutator result of Taussky and

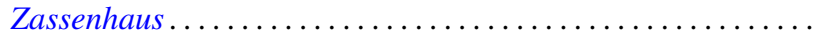

John Glen Marica and Steve Jerome Bryant, Unary algebras......

Edward Peter Merkes and W. T. Scott, On univalence of a continued fraction . . . . . . . 1361

Shu-Teh Chen Moy, Asymptotic properties of derivatives of stationary measures . . . . . 1371

John William Neuberger, Concerning boundary value problems . . . . . . . . . . . 1385

Edward C. Posner, Integral closure of differential rings . . . . . . . . . . . . . . . . . 1393

Marian Reichaw-Reichbach, Some theorems on mappings onto . . . . . . . . . . . . . 1397

Marvin Rosenblum and Harold Widom, Two extremal problems . . . . . . . . . . . . . . . . 1409

Morton Lincoln Slater and Herbert S. Wilf, A class of linear differential-difference

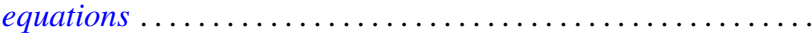

Charles Robson Storey, Jr., The structure of threads . . . . . . . . . . . . . . . . . . 1429

J. François Treves, An estimate for differential polynomials in $\partial / \partial z_{1},, \cdots, \partial / \partial z_{-} n \ldots \ldots 1447$

J. D. Weston, On the representation of operators by convolutions integrals . . . . . . . . 1453

James Victor Whittaker, Normal subgroups of some homeomorphism groups ......... 1469 